\title{
Cardiorenal Syndrome: An Unsolved Clinical Problem
}

\author{
Paula Martínez-Santos ${ }^{1}$ and Isidre Vilacosta ${ }^{2}$ \\ ${ }^{1}$ Hospital Universitario Fundación Alcorcón, Av. Budapest no. 1, Alcorcón, 28922 Madrid, Spain \\ ${ }^{2}$ Departmento de Cardiologia, Hospital Clínico San Carlos Profesor Martín Lagos, 28040 Madrid, Spain
}

Correspondence should be addressed to Paula Martínez-Santos, paulams1@hotmail.com

Received 20 August 2010; Revised 28 January 2011; Accepted 28 March 2011

Academic Editor: Anjay Rastogi

Copyright ( 2011 P. Martínez-Santos and I. Vilacosta. This is an open access article distributed under the Creative Commons Attribution License, which permits unrestricted use, distribution, and reproduction in any medium, provided the original work is properly cited.

The clinical relevance of the bidirectional cross-talk between heart and kidney is increasingly recognized. However, the optimal approach to the management of kidney dysfunction in heart failure remains unclear. The purpose of this article is to outline the most plausible pathophysiologic theories that attempt to explain the renal impairment in acute and chronic heart failure, and to review the current treatment strategies for these situations.

\section{Introduction}

Heart and kidney are inextricably linked to maintain homeostasis. Communication between these two organs occurs at multiple levels including the sympathetic nervous system (SNS), the renin-angiotensin-aldosterone system (RAAS), the antidiuretic hormone, endothelin, or the natriuretic peptides. The dysfunction of one of them contributes to the dysfunction of the other; renal dysfunction impairs cardiac performance, which again leads to further impairment of renal function. The term "cardiorenal syndrome" (CRS) was coined to define this situation, but a consensus of the diagnostic criteria has not been reached yet. Initially, it was characterized as a state in which therapy to relieve congestive heart failure (HF) symptoms was limited by further worsening renal function [1]. Although this definition does not accurately describe the complexity of its nature, it portrays a common situation in daily clinical practise. A broader definition of the CRS was developed by the Acute Dialysis Quality Initiative [2].

The CRS was classified into five categories, according to the underlying etiologies and the nature of concomitant cardiac and renal dysfunction (Table 1). Heart failure seems to be the primary failing organ in two of the five described features. CRS type 1 occurs when acute decompensated heart failure (ADHF) leads to acute kidney injury. CRS type 2 refers to the development of a progressive worsening of renal function (WRF) in the setting of chronic heart failure $(\mathrm{CHF})$. Both, acute and progressive development of renal dysfunction in patients with heart failure, have been associated with independently worse outcomes compared with preserved renal function [3-13]. Therefore, a precise understanding of the pathophysiology of this syndrome is needed to provide the rationale for management strategies.

\section{Pathophysiology}

The pathophysiology of the cardiorenal syndrome remains unclear but can be attributed to three main factors: lowcardiac output, elevation of both intra-abdominal and central venous pressures, and neurohormonal and inflammatory activation $[14,15]$. The terms "backward failure" and "forward failure" have been historically used to classify HF syndrome. Although not commonly used nowadays, this classification allows an intuitive approach to understand the underlying mechanisms of these forms of CRS. Forward failure implies arterial underfilling, which leads to a lowflow state. This appears to be one of the cornerstones in the development of CRS, but not the only one. Improvement in cardiac index did not always result in improved renal function. Multiple studies support this conclusion: The Evaluation Study of Congestive Heart Failure and Pulmonary Catheterization Effectiveness (ESCAPE) trial associated 
baseline kidney dysfunction (estimated glomerular filtration rate, -GFR-, $<60 \mathrm{~mL} / \mathrm{min}$ ) at admission and at discharge with an increased risk of death and rehospitalization [16]. Patients randomized to the group, in which therapy was guided by clinical assessment and a pulmonary artery catheter (PAC), presented significantly less deterioration of kidney function, compared with a therapy based on clinical assessment alone, but this did not imply an improvement in clinical outcomes in patients with baseline CKD. Incidence of WRF during hospitalization (serum creatinine $>0.3 \mathrm{mg} / \mathrm{dL}$ ) was similar in both arms, and was not associated to increased outcomes of death or rehospitalization. Among hemodynamic parameters measured in the PAC arm, only right atrial pressure correlated weakly with baseline serum creatinine $(r=0.165$, $P=.03$ ). Similar results were obtained by Mullens et al. [17]. They studied 145 patients admitted with ADHF and treated with intensive medical therapy guided by pulmonary artery catheter. Patients who developed WRF did not have a lower cardiac index on admission or at discharge when compared with those without WRF. The mean baseline cardiac index was significantly greater in subjects who developed WRF versus those who did not $\left(2.00 \pm 0.8 \mathrm{l} / \mathrm{min} / \mathrm{m}^{2}\right.$ versus 1.8 $\left.\pm 0.4 \mathrm{l} / \mathrm{min} / \mathrm{m}^{2}, P=.008\right)$. At follow-up, the mean cardiac index and the central venous pressure remained superior $(2.7$ $\pm 0.7 \mathrm{l} / \mathrm{min} / \mathrm{m}^{2}$ versus $2.4 \pm 0.5 \mathrm{l} / \mathrm{min} / \mathrm{m}^{2}, P=.01$ and $11 \pm$ $8 \mathrm{~mm} \mathrm{Hg}$ versus $8 \pm 5 \mathrm{~mm} \mathrm{Hg}, P=.04$, resp.) in subjects who developed WRF.

These findings support the hypothesis that there must be another mechanism that contributes to renal impairment in heart failure. Rising renal venous pressure limits urine formation and renal flow. Several mechanisms have been proposed to explain this situation. Backward failure implies that systemic venous congestion also affects renal venous pressure and function (congestive kidney failure), by direct hypoxic damage or through RAAS/SNS stimulation way.

A substudy of the Studies of Left Ventricular Dysfunction (SOLVD) established the prognostic implication of jugular venous pressure on patients with CHF [18]. Patients with increased venous pressure had a significantly higher serum creatinine level $(115 \pm 27$ versus $106 \pm 27 \mu \mathrm{mol} / \mathrm{L})$. Interestingly, Mullens et al. also described that patients who developed WRF had greater central venous pressure on admission (18 $\pm 7 \mathrm{~mm} \mathrm{Hg}$ versus $12 \pm 6 \mathrm{~mm} \mathrm{Hg}, P=.001)$ and after intensive medical therapy $(11 \pm 8 \mathrm{~mm} \mathrm{Hg}$ versus $8 \pm 5 \mathrm{~mm} \mathrm{Hg}, P=.04$ ) [17]. The development of WRF occurred less frequently in patients who achieved a central venous pressure $<8 \mathrm{~mm} \mathrm{Hg}$. Damman et al. evaluated right atrial pressure and cardiac index by right heart catheterisation, in 51 patients with cardiac dysfunction, secondary to pulmonary hypertension [19]. In a multivariate analysis, low renal blood flow and high right atrial pressure were independently associated with lower GFR.

Intra-abdominal pressure has been considered an alternative pathway to explain how decompensated HF may lead to WRF. Increased abdominal pressure may lead to renal impairment by a "compressing effect" in renal parenchyma. Hence, elevated intra-abdominal pressure (defined as $>8 \mathrm{~mm} \mathrm{Hg}$ ) has been associated with significantly lower GFR compared with those with normal IAP in patients
TABLE 1: Cardiorenal syndrome: classification.

\begin{tabular}{ll}
\hline CRS type 1 & $\begin{array}{l}\text { Development of acute kidney injury in the setting } \\
\text { of a sudden deterioration of heart function }\end{array}$ \\
CRS type 2 & $\begin{array}{l}\text { Progressive renal dysfunction in the setting of } \\
\text { chronic cardiac dysfunction }\end{array}$ \\
CRS type 3 & $\begin{array}{l}\text { Abrupt and primary worsening of renal function } \\
\text { leads to acute heart failure }\end{array}$ \\
CRS type 4 & $\begin{array}{l}\text { Primary chronic kidney disease contributes to the } \\
\text { progressive development of chronic heart failure } \\
\text { Combined cardiac and renal dysfunction caused } \\
\text { by a systemic illness }\end{array}$ \\
\hline
\end{tabular}

TABLE 2: Summary of recommendations for clinical practice.

(i) Search for reversible causes: concomitant medications, hypovolemia, hypotension, and urinary tract obstruction

(ii) Loop diuretics are useful to alleviate congestive symptoms but should be used with caution: check renal function and serum electrolytes closely

(iii) ACEI, ARA II, and aldosterone antagonists should be add, in case of heart failure and systolic dysfunction: check renal function and serum electrolytes closely

(iv) Ultrafiltration may be considered refractory to diuretics in symptomatic patients

(v) Correcting anemia should be considered in cardiorenal syndrome type 2

with advanced decompensated HF (mean LVEF 19\%) [20]. These authors also studied the effect of mechanical fluid removal to reduce IAP in patients with ADHF, showing a strong correlation $(r=0.77, P<.001)$ was observed between improved renal function in patients reduction in IAP and with baseline elevated IAP [21].

In any case, hemodynamic changes do not fully explain the whole cardiorenal connection. Interventions focused on the interactions of the networks which link both systems (RAAS, imbalance between reactive oxygen species and nitric oxide, sympathetic nervous system and inflammation processes) might help to control the progression of the CRS [22].

\section{Management of Worsening Renal Function in the Setting of Acute Decompensated Heart Failure}

Despite its common pathophysiology, each CRS's type embraces a broad spectrum of clinical features. Therefore, therapies should be adapted to each single patient's own situation (Table 2).

Loop diuretics are first-line agents to alleviate congestive symptoms [23]. Although their use is widespread, there is little evidence of their influence in managing CRS. Their use may be associated with electrolyte abnormalities, further neurohormonal activation and worsening renal function. In addition, they may increase the risk to develop adverse effects of concomitant medications, such as angiotensin converting enzyme inhibitors (ACEI), angiotensin II receptor blockers 
(ARB), or spironolactone. Hence, the first problem to deal with is to strike a balance between removing volume to relieve congestion without stimulating adverse effects.

There is lack of evidence in the literature about this topic, not only in AHF, but also in CKD patients, as they are systematically excluded from randomized trials. High-dose administration of intravenous loop diuretics has been associated with worse outcomes in HF patients [24, 25]. Results of the Dose Optimization Strategy Evaluation (DOSE) trial, recently presented at the American College of Cardiology (ACC) 2010 Scientific Sessions, may highlight this matter [26]. It included acute heart-failure patients with a prior diagnosis of chronic heart failure (CHF) and daily outpatient use of oral loop diuretics ( $80 \mathrm{mg}$ to $240 \mathrm{mg}$ ) for at least one month. Patients with serum creatinine $>3 \mathrm{mg} / \mathrm{dL}$ were excluded. Patients were randomized to either high dose $(2.5 \times$ their daily chronic oral furosemide dose given iv) or low dose (their daily chronic oral furosemide dose given iv) and were also randomized to dosing via intravenous bolus or continuous infusion. Median baseline creatinine was $1.5 \mathrm{mg} / \mathrm{dL}$. The primary endpoints were symptom resolution and change in serum creatinine from admission to 72 hours. There were no significant differences among the different dosing strategies for any of the two endpoints. The high-dose strategy showed greater symptom improvement, $(P=.06)$, but was also associated with mild increases in creatinine levels, defined as $a>0.3 \mathrm{mg} / \mathrm{dL}$ rise in creatinine. There were no differences among groups for death or rehospitalization outcomes. Results of this trial suggest that, apparently, an overaggressive use of loop diuretic is as safe as a conservative treatment. If the response to loop diuretics is inadequate, a thiazide should be added in a dose determined according to the patient's renal function. A synergistic response can result in profound diuresis. These patients should, therefore, be followed closely to prevent volume, magnesium, and potassium depletion. Again, this empirical management, which results to be effective in daily practise, has not been tested.

Vasodilators, such as intravenous nitroglycerin, are recommended at an early stage for AHF patients without hypotension or serious obstructive valvular disease [23]. The reduction in venous pressure may improve transrenal blood flow while protecting renal function, but doses that decrease blood pressure may cause a decline in renal perfusion and further activation of the RAAS. Although it has been published that isosorbide dinitrate should have a beneficial effect in patients with AHF [27], no randomized controlled studies have been carried out to evaluate its role neither in cardiorenal syndrome nor even in AHF. Nesiritide is a recombinant analogue of human brain natriuretic peptide for exogenous administration. The ASCEND trial (A Study testing the Effectiveness of Nesiritide in Patients with Acute Decompensated Heart Failure) enrolled 7,141 patients with severe HF, to determine whether nesiritide was superior to placebo in reducing the HF related hospitalization rate or all cause mortality at 30-days and improvement in dyspnea at six or 24 hours. Patients were randomly assigned to continuous intravenous nesiritide or placebo plus standard treatment for 30 days. Compared with placebo, nesiritide was not associated with a reduction in 30 day death or HF rehospitalization (10.1\% versus $9.4 \% ; P=.31$ ). Data from ASCEND-HF showed no association between nesiritide and reduced renal function [28]. Vasopressin antagonists selectively inhibit the $\mathrm{V} 2$ receptor of renal distal tubules and collecting duct, increasing aquaresis and serum sodium in those who are hyponatremic. Safety and efficacy of these agents have been tested in several trials in patients with AHF during the acute phase. Although they have not proven long-term benefit on clinical outcomes, they could have a favorable effect on renal hemodynamics [29].

Adenosine A1 receptor antagonists improve renal blood flow and increase sodium excretion, by enhancing natriuresis, with preserving GFR, in combination with furosemide. An ongoing trial (A Study of the Selective Al Adenosine Receptor Antagonist KW-3902 for Patients Hospitalized With Acute HF and Volume Overload to Assess Treatment Effect on Congestion and Renal Function, PROTECT-2 study) is evaluating its application in ADHF patients.

Ultrafiltration helps to remove the volume overload in symptomatic patients refractory to diuretics. The Ultrafiltration versus Intravenous Diuretics for Patients Hospitalized for Acute Decompensated Congestive Heart Failure (UNLOAD) trial demonstrated a greater weight loss in ultrafiltration group, as well as lower rehospitalization rates and emergency department visits, compared to a diureticbased strategy [30].

Inotropic agents should be considered in patients with low-output states in the presence of signs of hypoperfusion or congestion despite the use of vasodilators and/or diuretics [23]. Dobutamine has been associated to an increase in renal blood flow, proportional to the increase in cardiac index. Low-dose dopamine has also been associated with a theoretical effect on renal blood flow. Although it has been proposed that an improvement in cardiac output might lead to preserving renal function, this widespread empirical therapy has not been tested in randomized trials. Only milrinone and levosimendan have been evaluated in this situation, and none of them have demonstrated an improvement in renal function $[15,31]$.

\section{Management of Renal Dysfunction in Chronic Heart Failure}

Mechanisms which lead to a progressive renal impairment in patients with $\mathrm{CHF}$ are still unclear. Several factors may contribute to this situation, including hemodynamic changes, diuretic's side effects, or a microvascular damage secondary to a concomitant illness (such as hypertension or diabetes).

Although many pharmacological and no pharmacological therapies have proven to be an advantage on survival in HF, their prescription is often limited by the fear to develop complications attributable to WRF. The results of the Registry to Improve the Use of EvidenceBased Heart Failure Therapies in the Outpatient Setting (IMPROVE HF) have been recently published [32]. The aim of this prospective study was to evaluate the adherence 
to AHA/ACC guidelines recommendations in the management of chro-nic heart failure (ACEIs/ARB, $\beta$-blockers, aldoste-rone antagonists, cardiac resynchronization therapy, implantable cardioverter defibrillator, anticoagulation if atrial fibrillation/flutter, and patient education) and to determine the influence of renal dysfunction in applying these therapies. It included 13,164 nonhospitalized patients with severe systolic dysfunction (LVEF < 35\%). Patients were classified into four groups, according to their CKD stage. Mean LVEF was around $25 \%$ in all groups. About $52 \%$ of the patients had a GFR $<60 \mathrm{~mL} / \mathrm{min} / 1.73 \mathrm{~m}^{2}$. The use of ACEI/ARB $(87.4 \%$ in patients with GFR $>90 \mathrm{~mL} / \mathrm{min} / 1.73 \mathrm{~m}^{2}$ versus $57.9 \%$ in patients with GFR $\left.<29 \mathrm{~mL} / \mathrm{min} / 1.73 \mathrm{~m}^{2}, P<.001\right), \beta$-blockers $(90.4 \%$ in patients with GFR $>90 \mathrm{~mL} / \mathrm{min} / 1.73 \mathrm{~m}^{2}$ versus $86.2 \%$ in patients with GFR $<29 \mathrm{~mL} / \mathrm{min} / 1.73 \mathrm{~m}^{2}$ ), and aldosterone antagonists was significantly lower in patients with a higher stage of CKD. Multivariate analysis showed that the severity of $\mathrm{CKD}$ was an independent predictor of adherence to ACEI/ARB (HR 0.94; CI 95\% 0.88-0.99; $P=.018$ ), but not in the other recommended interventions.

Treatment with ACEI improves ventricular function (as evidenced by an increased ejection fraction and decreased ventricular size) and patient well-being, reduces hospital admission for worsening HF, and increases survival. This therapy should be used in all patients with symptomatic $\mathrm{HF}$ and a $\mathrm{LVEF}<40 \%$ [23]. As it is common to observe a significant increase in the serum creatinine concentration $(>0.3 \mathrm{mg} / \mathrm{dL}$ ) within the initiation of treatment with ACEI, renal function should be closely monitored. ESC Guidelines of management of HF accept a 50\% increase in creatinine serum level from baseline or an absolute concentration of $3 \mathrm{mg} / \mathrm{dL}$, whichever is lower. If creatinine rises between 3$3.5 \mathrm{mg} / \mathrm{dL}$, ESC guidelines recommend to halve dose of ACEI. Treatment with ACEI must be interrupted if creatinine serum concentration rises above $3.5 \mathrm{mg} / \mathrm{dL}$. ARB may be considered as an alternative in patients who do not tolerate ACEI. The influence on cardiorenal protection of RAAS dual blockade, when an ARB is used in conjunction with an ACEI, has also been analyzed in several trials. Among patients with $\mathrm{HF}$, combination therapy was associated with further impairment in kidney function [33]. An aldosterone antagonist should be added to treatment in symptomatic patients with HF and an LVEF < 35\% [23]. Similar to ACEI, renal function should be closely monitored. If creatinine rises above $2.5 \mathrm{mg} / \mathrm{dL}$ (or potassium > $5.5 \mathrm{mmol} / \mathrm{L}$ ), ESC guidelines suggest to halve the spironolactone or eplerenone doses. When serum creatinine is $>3 \mathrm{mg} / \mathrm{dL}$ (or potassium $>$ $6 \mathrm{mmol} / \mathrm{L}$ ), treatment with aldosterone antagonists must be discontinued.

The Canadian Cardiovascular Society includes among its recommendations some options of management for these patients. Thus, renal function must be checked daily in patients with heart failure and increasing serum creatinine more than 30\% from baseline, and ACEI, ARB, and aldosterone dose should be reduced until renal function stabilizes. In oliguric HF patients treatment with diuretics, ACEI, ARB, or aldosterone should be reviewed daily. Routine use of ACE inhibitors, ARBs or spironolactone in the setting of severe renal dysfunction (serum creatinine levels greater than $250 \mu \mathrm{mol} / \mathrm{L}$ or an increase of more than $50 \%$ from baseline) is not routinely recommended [34].

Despite these recommendations, it is important to emphasize that serum creatinine concentration is not an accurate measure of GFR. Creatinine serum levels vary according to gender, person's size, and muscle mass, and this must be taken into account. The most common formula for calculating the GFR has not been validated in acute renal failure, but there is on increasing interest in new renal biomarkers for the diagnosis and classification of CRS, such as cystatin $\mathrm{C}$ or the neutrophil gelatinase-associated lipocalin [35].

Anemia is a frequent and multifactorial finding in both $\mathrm{CKD}$ and $\mathrm{CHF}$. Its prevalence is similar among patients with preserved and depressed LVEF. Neither ACC/AHA nor ESC guidelines establish the correction of anemia as a systematic target in patients with HF, although it has been associated with poor clinical outcomes. Even though there is actually no definitive evidence to the optimum approach for the management of anemia in patients with CHF which develop a progressive renal dysfunction (cardiorenal syndrome type 2), a pragmatical strategy for anemia correction, based on CKD guidelines and HF trials, has been proposed [36]. Because of the adverse cardiovascular effects of higher hematocrit in CKD trials, authors suggest a target hemoglobin of 10$12 \mathrm{~g} / \mathrm{dL}$ at clinical practise. If $\mathrm{Hb}$ drops below $10 \mathrm{~g} / \mathrm{dL}$, iron deficiency should be excluded before starting therapy with erythropoiesis stimulating agents. Nonetheless, patients with HF might benefit from more aggressive anemia correction in view of the results of several cardiology trials [36-39].

\section{Conclusion}

Cardiorenal syndrome implies several interrelated mechanisms in patients with heart failure. The appropriate strategy to take care of these patients remains unclear, both in acute and chronic clinical situations. In accordance with the most plausible underlying pathophysiological mechanisms, treatment targets should be oriented toward an adequate intravascular volume management and to ensure a proper renal perfusion. Although there are encouraging advances around this unsolved clinical problem, further investigation should consider the progressive inclusion of patients with advanced renal impairment to allow a better understanding of cardiorenal syndrome.

\section{References}

[1] National Heart, Lung, and Blood Institute Working Group, Cardiorenal Connections in Heart and Cardiovascular Disease, National Heart, Lung, and Blood Institute, 2007.

[2] C. Ronco, M. Haapio, A. A. House, N. Anavekar, and R. Bellomo, "Cardiorenal Syndrome," Journal of the American College of Cardiology, vol. 52, no. 19, pp. 1527-1539, 2008.

[3] S. S. Gottlieb, W. Abraham, J. Butler et al., "The prognostic importance of different definitions of worsening renal function in congestive heart failure," Journal of Cardiac Failure, vol. 8, no. 3, pp. 136-141, 2002. 
[4] H. M. Krumholz, Y. T. Chen, V. Vaccarino et al., "Correlates and impact on outcomes of worsening renal function in patients $\geq 65$ years of age with heart failure," American Journal of Cardiology, vol. 85, no. 9, pp. 1110-1113, 2000.

[5] J. T. Heywood, G. C. Fonarow, M. R. Costanzo, V. S. Mathur, J. R. Wigneswaran, and J. Wynne, "High prevalence of renal dysfunction and its impact on outcome in 118,465 patients hospitalized with acute decompensated heart failure: a report from the ADHERE database," Journal of Cardiac Failure, vol. 13, no. 6, pp. 422-430, 2007.

[6] M. R. Cowie, M. Komajda, T. Murray-Thomas, J. Underwood, and B. Ticho, "Prevalence and impact of worsening renal function in patients hospitalized with decompensated heart failure: results of the prospective outcomes study in heart failure (POSH)," European Heart Journal, vol. 27, no. 10, pp. 1216-1222, 2006.

[7] D. Logeart, J. Y. Tabet, L. Hittinger et al., “Transient worsening of renal function during hospitalization for acute heart failure alters outcome," International Journal of Cardiology, vol. 127, no. 2, pp. 228-232, 2008.

[8] M. Metra, S. Nodari, G. Parrinello et al., "Worsening renal function in patients hospitalised for acute heart failure: clinical implications and prognostic significance," European Journal of Heart Failure, vol. 10, no. 2, pp. 188-195, 2008.

[9] M. G. Shlipak, G. L. Smith, S. S. Rathore, B. M. Massie, and H. M. Krumholz, "Renal function, digoxin therapy, and heart failure outcomes: evidence from the digoxin intervention group trial," Journal of the American Society of Nephrology, vol. 15, no. 8, pp. 2195-2203, 2004.

[10] S. M. Bagshaw, D. N. Cruz, N. Aspromonte et al., "Epidemiology of cardio-renal syndromes: workgroup statements from the 7th ADQI Consensus Conference," Nephrology Dialysis Transplantation, vol. 25, no. 5, pp. 1406-1416, 2010.

[11] A. Al-Ahmad, W. M. Rand, G. Manjunath et al., "Reduced kidney function and anemia as risk factors for mortality in patients with left ventricular dysfunction," Journal of the American College of Cardiology, vol. 38, no. 4, pp. 955-962, 2001.

[12] H. L. Hillege, A. R. J. Girbes, P. J. De Kam et al., "Renal function, neurohormonal activation, and survival in patients with chronic heart failure," Circulation, vol. 102, no. 2, pp. 203-210, 2000.

[13] N. A. Khan, I. Ma, C. R. Thompson et al., "Kidney function and mortality among patients with left ventricular systolic dysfunction," Journal of the American Society of Nephrology, vol. 17, no. 1, pp. 244-253, 2006.

[14] W. H. W. Tang and W. Mullens, "Cardiorenal syndrome in decompensated heart failure," Heart, vol. 96, no. 4, pp. 255260, 2010 .

[15] J. S. Bock and S. S. Gottlieb, "Cardiorenal syndrome: new perspectives," Circulation, vol. 121, no. 23, pp. 2592-2600, 2010.

[16] A. Nohria, V. Hasselblad, A. Stebbins et al., "Cardiorenal Interactions. Insights From the ESCAPE Trial," Journal of the American College of Cardiology, vol. 51, no. 13, pp. 1268-1274, 2008.

[17] W. Mullens, Z. Abrahams, G. S. Francis et al., "Importance of venous congestion for worsening of renal function in advanced decompensated heart failure," Journal of the American College of Cardiology, vol. 53, no. 7, pp. 589-596, 2009.

[18] M. H. Drazner, J. E. Rame, L. W. Stevenson, and D. L. Dries, "Prognostic importance of elevated jugular venous pressure and a third heart sound in patients with heart failure," New England Journal of Medicine, vol. 345, no. 8, pp. 574-581, 2001.

[19] K. Damman, G. Navis, T. D. J. Smilde et al., "Decreased cardiac output, venous congestion and the association with renal impairment in patients with cardiac dysfunction," European Journal of Heart Failure, vol. 9, no. 9, pp. 872-878, 2007.

[20] W. Mullens, Z. Abrahams, H. N. Skouri et al., "Elevated intraabdominal pressure in acute decompensated heart failure. A potential contributor to worsening renal function?" Journal of the American College of Cardiology, vol. 51, no. 3, pp. 300-306, 2008.

[21] W. Mullens, Z. Abrahams, G. S. Francis, D. O. Taylor, R. C. Starling, and W. H. W. Tang, "Prompt reduction in intraabdominal pressure following large-volume mechanical fluid removal improves renal insufficiency in refractory decompensated heart failure," Journal of Cardiac Failure, vol. 14, no. 6, pp. 508-514, 2008.

[22] L. G. Bongartz, M. J. Cramer, P. A. Doevendans, J. A. Joles, and B. Braam, "The severe cardiorenal syndrome: "Guyton revisited'”' European Heart Journal, vol. 26, no. 1, pp. 11-17, 2005.

[23] K. Dickstein, A. Cohen-Solal, G. Filippatos et al., "ESC Guidelines for the diagnosis and treatment of acute and chronic heart failure 2008. The Task Force for the Diagnosis and Treatment of Acute and Chronic Heart Failure 2008 of the European Society of Cardiology. Developed in collaboration with the Heart Failure Association of the ESC (HFA) and endorsed by the European Society of Intensive Care Medicine (ESICM)," European Journal of Heart Failure, vol. 10, no. 10, pp. 933-989, 2008.

[24] M. Domanski, J. Norman, B. Pitt, M. Haigney, S. Hanlon, and E. Peyster, "Diuretic use, progressive heart failure, and death in patients in the Studies Of Left Ventricular Dysfunction (SOLVD)," Journal of the American College of Cardiology, vol. 42, no. 4, pp. 705-708, 2003.

[25] V. Hasselblad, W. G. Stough, M. R. Shah et al., "Relation between dose of loop diuretics and outcomes in a heart failure population: results of the ESCAPE Trial," European Journal of Heart Failure, vol. 9, no. 10, pp. 1064-1069, 2007.

[26] The Diuretic Optimization Strategies Evaluation (DOSE) Study: a Randomized, Double Blind, Placebo-controlled Trial Of Diuretic Strategies In Acute Decompensated Heart Failure, 2010, NCT00577135, http://www.clinicaltrials.gov/.

[27] G. Cotter, E. Metzkor, E. Kaluski et al., "Randomised trial of high-dose isosorbide dinitrate plus low-dose furosemide versus high-dose furosemide plus low-dose isosorbide dinitrate in severe pulmonary oedema," Lancet, vol. 351, no. 9100, pp. 389-393, 1998.

[28] A Study Testing the Effectiveness of Nesiritide in Patients With Acute Decompensated Heart Failure, NCT00475852, http:// www.clinicaltrials.gov/.

[29] J. Rossi, M. Bayram, J. E. Udelson et al., "Improvement in hyponatremia during hospitalization for worsening heart failure is associated with improved outcomes: insights from the Acute and Chronic Therapeutic Impact of a Vasopressin Antagonist in Chronic Heart Failure (ACTIV in CHF) trial," Acute Cardiac Care, vol. 9, no. 2, pp. 82-86, 2007.

[30] M. R. Costanzo, M. T. Saltzberg, M. Jessup, J. R. Teerlink, and P. A. Sobotka, "Ultrafiltration is associated with fewer rehospitalizations than continuous diuretic infusion in patients with decompensated heart failure: results from UNLOAD," Journal of Cardiac Failure, vol. 16, no. 4, pp. 277-284, 2010. 
[31] S. R. Goldsmith, F. Brandimarte, and M. Gheorghiade, "Congestion as a therapeutic target in acute heart failure syndromes," Progress in Cardiovascular Diseases, vol. 52, no. 5, pp. 383-392, 2010.

[32] J. T. Heywood, G. C. Fonarow, C. W. Yancy et al., "Influence of renal function on the use of guideline-recommended therapies for patients with heart failure," American Journal of Cardiology, vol. 105, no. 8, pp. 1140-1146, 2010.

[33] M. Arici and Y. Erdem, "Dual blockade of the reninangiotensin system for cardiorenal protection: an update," American Journal of Kidney Diseases, vol. 53, no. 2, pp. 332345, 2009.

[34] J. M. O. Arnold, J. G. Howlett, P. Dorian et al., "Canadian Cardiovascular Society Consensus Conference recommendations on heart failure update 2007. Prevention, management during intercurrent illness or acute decompensation, and use of biomarkers," Canadian Journal of Cardiology, vol. 23, no. 1, pp. 21-45, 2007.

[35] D. N. Cruz, S. Soni, L. Slavin, C. Ronco, and A. Maisel, "Biomarkers of cardiac and kidney dysfunction in cardiorenal syndromes," Contributions to Nephrology, vol. 165, pp. 83-92, 2010.

[36] A. Kazory and E. A. Ross, "Anemia: the point of convergence or divergence for kidney disease and heart failure?" Journal of the American College of Cardiology, vol. 53, no. 8, pp. 639-647, 2009.

[37] J. S. Golden, C. C. Fallick, S. R. Josephson et al., "Baseline hematocrit predicts clinical outcomes in heart failure," Circulation, vol. 106, supplement 2, p. 3358A, 2002.

[38] H. Reinecke, T. Trey, J. Wellmann et al., "Haemoglobin-related mortality in patients undergoing percutaneous coronary interventions," European Heart Journal, vol. 24, no. 23, pp. 2142-2150, 2003.

[39] R. Sharma, D. P. Francis, B. Pitt, P. A. Poole-Wilson, A. J. S. Coats, and S. D. Anker, "Haemoglobin predicts survival in patients with chronic heart failure: a substudy of the ELITE II trial," European Heart Journal, vol. 25, no. 12, pp. 1021-1028, 2004. 


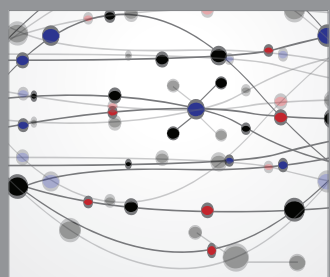

The Scientific World Journal
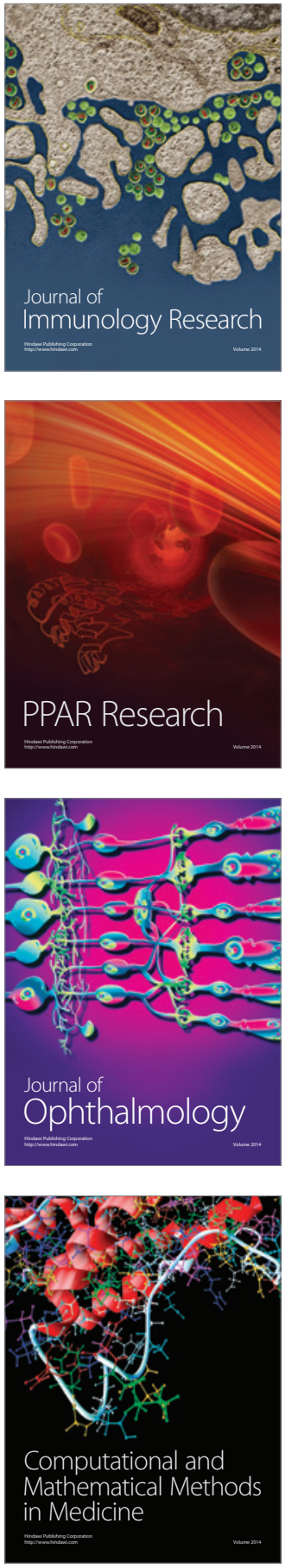

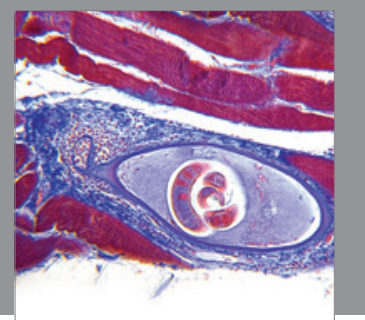

Gastroenterology

Research and Practice
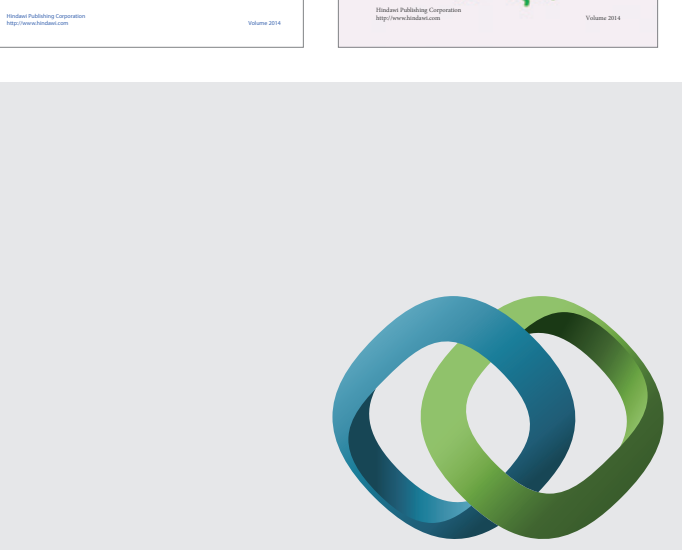

\section{Hindawi}

Submit your manuscripts at

http://www.hindawi.com
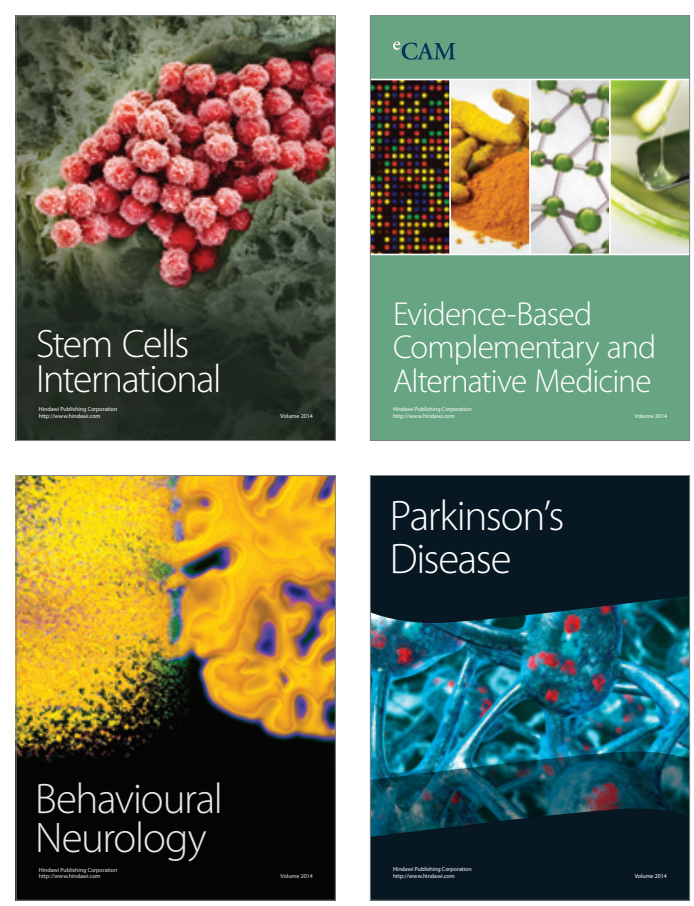

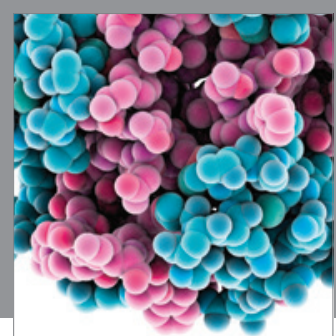

Journal of
Diabetes Research

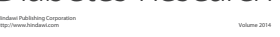

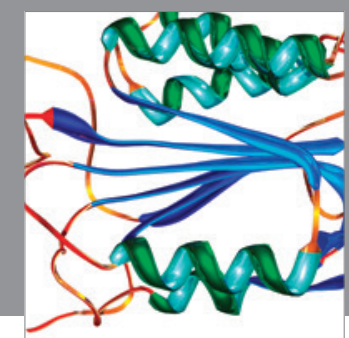

Disease Markers
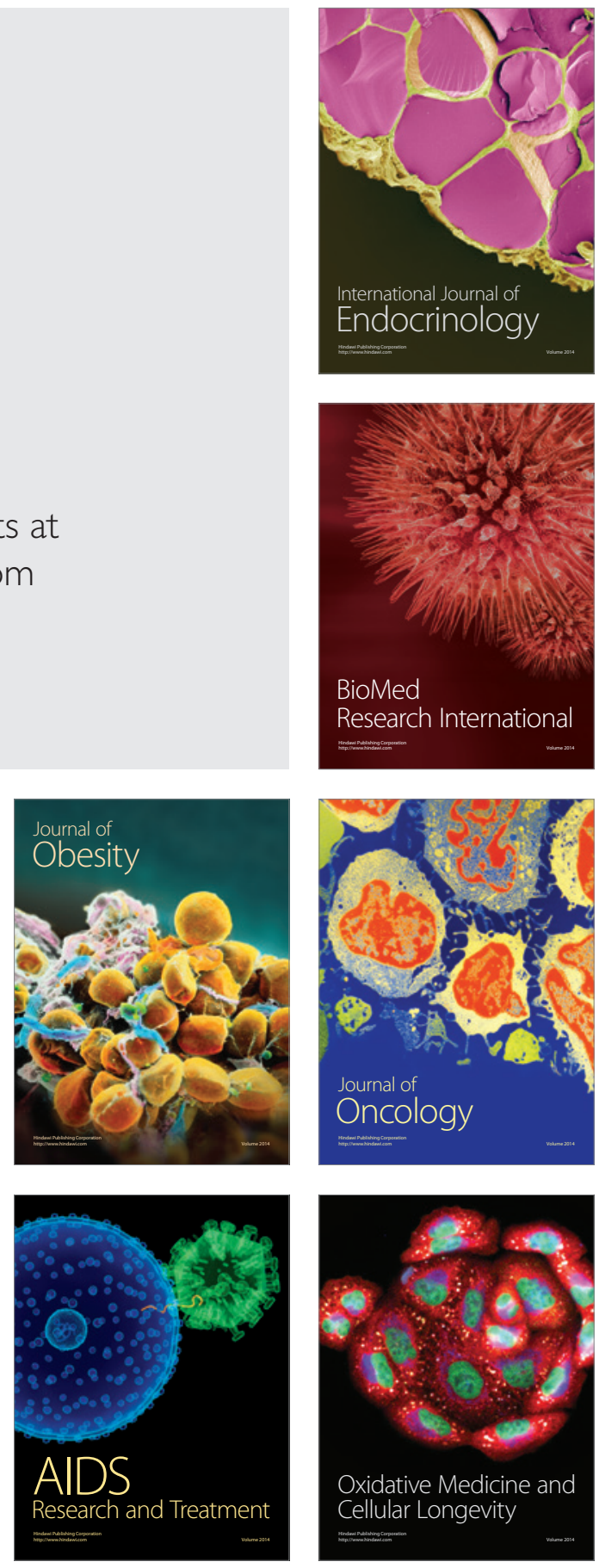